\title{
DOPPLER TRANSCRANIANO NA PRÁTICA NEUROLÓGICA
}

\author{
Viviane Flumignan Zétola', Marcos Christiano Lange', Juliano A. Muzzio², \\ Ivo Marchioro², Edison Mattos Nóvak³ , Lineu C. Werneck ${ }^{4}$
}

\begin{abstract}
RESUMO - O Doppler transcraniano (DTC) é um método relativamente novo, descrito em 1982 por Rune Aaslid e introduzido no Brasil em 1992 por Roberto Hirsch, na Universidade de São Paulo. O serviço de Neurologia do Hospital de Clínicas da Universidade Federal do Paraná (HC-UFPR) disponibilizou esse exame em 2001, com divulgação das principais vantagens do método no diagnóstico, seguimento e manejo terapêutico de pacientes com suspeita ou portadores de doença cerebrovascular. $\mathrm{O}$ objetivo deste trabalho é demonstrar como o DTC vem sendo utilizado em nosso meio e quais são as especialidades médicas que vêm absorvendo o auxílio do método. Embora diretrizes como a publicada pela Academia Americana de Neurologia em 2004 validem as indicações do DTC, estabelecendo especificidade e sensibilidade do método, verificamos que a solicitação do exame em nosso meio depende da divulgação do serviço, da implementação de linhas de pesquisa e, finalmente, do reconhecimento de sua utilidade na prática diária.
\end{abstract}

PALAVRAS-CHAVE: Doppler transcraniano.

\begin{abstract}
Transcranial Doppler in the neurological practice
ABSTRACT - Transcranial Doppler (TCD) was described in 1982 by Rune Aaslid and introduced in Brazil in 1992 by Roberto Hirsh in the University of São Paulo. Since the acquisition of TCD by Neurology Division of Hospital de Clínicas of Universidade Federal do Paraná (HC-UFPR) in 2001, the knowledge about TCD clinical utility has been increasing for diagnosis, follow-up and management of patients with cerebrovascular diseases. The objective of this study is to describe the current TCD indications and medical specialties indicators in our hospital. Although guidelines as published by the American Academy of Neurology establish values for TCD study with method's specificity and sensibility, we realize that the utility in our center depends on our efforts to show method's beneficial in various clinical settings.
\end{abstract}

KEY WORDS: Transcranial Doppler.

O Doppler transcraniano (DTC) é método relativamente novo, não-invasivo, que utiliza a técnica do ultra-som para medir indiretamente o fluxo nas porções proximais das principais artérias intracranianas ( $p$ e rtencentes ao polígono de Willis), oferecendo informações dinâmicas da circulação cerebral. O exame foi descrito em 1982 por Rune Aaslid e, desde então, vem sendo pro g ressivamente introduzido na prática clínica mundial ${ }^{1}$. No Brasil, a Universidade de São Paulo, em 1992, foi a primeira instituição a disponibilizar o método tendo como técnico-pioneiro o médico neurologista Roberto Hirsch. O DTC necessita de ope- rador com treinamento e experiência tanto para realização quanto para a interpretação dos resultados, bem como tem a dificuldade da barreira óssea que, por vezes, não permite a realização completa do exame. As principais vantagens são sua portabilidade, que viabiliza a realização em unidades intensivas, à beira do leito e durante ciru rgias ou procedimentos, e a ausência de invasibilidade ou toxicidade, o que possibilita a repetição do exame, permitindo o acompanhamento hemodinâmico do paciente. Recente publicação questiona uma possível ação terapêutica em casos de trombólise endovenosa². Embora as prin-

Seniço de Neurologia do Hospital de Clínicas da Universidade Federal do Paraná, Curitiba PR, Brasil (HCUFPR) e Hospital XV, Curitiba PR, Brasil: ' 1 Médico Neurologista HCUFPR; ${ }^{2}$ Médico Neurologista, Hospital XV; ${ }^{3}$ Professor Assistente HCUFPR; ${ }^{4}$ P rofessor Titular HCUFPR.

Recebido 4 Abril 2005, recebido na forma final 14 Julho 2005. Aceito 21 Setembro 2005.

Dra. Viviane Flumignan Zétola - Serviço de Neurologia - Hospital de Clínicas - Rua General Came iro 181 / sala 1236 - $80060-900$ Curitiba PR - Brasil. E-mail: viviane@flumignano.com 
cipais indicações constituam a avaliação diagnóstica e o controle terapêutico de pacientes com doenças cerebrovasculares, linhas de pesquisa preventivas (anemia falciforme), investigativa (pesquisa de shunt direita-esquerda) e de monitoração de procedimentos endovasculares/cirúrgicos (cardíaca e carotídea) vêm sendo fortemente estabelecidas ${ }^{3-5}$.

O Serviço de Neurologia do Hospital de Clínicas da Universidade Federal do Paraná (HC-UFPR) disponibilizou esse exame em agosto de 2001 e, desde então, vem realizando exames de rotina ambulatorial e emergencial. Houve necessidade de divulgar o método bem como estimular linhas de pesquisa com o objetivo de validação da metodologia ${ }^{6,7}$. Analisaremos o aspecto evolutivo da utilização do DTC em nosso meio, bem como descreveremos brevemente as diretrizes estabelecidas pela Academia Americana de Neurologia (AAN) ${ }^{8}$.

\section{MÉTODO}

Foram revisados 1120 laudos de exames de DTC realiza dos no Serviço de Neurologia do HC-UFPR entre os anos de 2001 e 2004. Todos os exames foram realizados e/ou supervisionados por neurologista experiente na utilização do método. Os exames foram realizados com aparelho multicanal com transdutor de $2 \mathrm{MHz}$ (Rimed Trans-link 9900 TCD, Herdiya, Israel). Analisamos os seguintes itens: 1) especialidade médica solicitante do exame; 2 ) indicação clínica; e 3) correlação positiva entre a hipótese e o resultado do exame.

\section{RESULTADOS}

E $n$ t re os 1120 exames analisados, 1008 pacientes realizaram apenas um exame, os demais realizaram entre dois e seis exames de seguimento. A média de idade dos pacientes foi 61,5 anos variando entre $1 \mathrm{e}$ 93 anos, sendo $60 \%$ do sexo feminino. Observou-se aumento prog ressivo do número de exames no decorrer dos quatro anos: em 2001 foram realizados apenas 30 exames (início em agosto daquele ano); em 2002, foram realizados 298 exames; em 2003 foram realizados 387, e finalmente, de janeiro até setembro de 2004 foram realizados 405 exames. A dificuldade de insonação através da janela temporal ocorreu em 118 (11\%) casos.

As especialidades que solicitaram com maior freqüência o exame foram a neurologia e neurocirurgia, analisadas em conjunto devido à semelhança das indicações. Em seguida vieram a otorrinolaringologia, a terapia intensiva e a cardiologia. Especialidades como ciru rgia cardíaca, clínica geral, ciru rgia vascular e pediatria vêm utilizando esse método mais recentemente (Tabela).

Aproximadamente $78 \%$ dos exames solicitados tiveram como indicação insuficiência vertebrobasilar (IVB) posicional (437), monitoração de microembolias (172), investigação de acidente vascular cerebral (AVC) (160) e investigação de vasoespasmo pós-hemorragia subaracnóidea espontânea (113). Outras indicações como morte encefálica, estudo de re s e rva funcional

Tabela. Especialidades médicas solicitantes de Doppler transcra niano entre 2001 e 2004.

\begin{tabular}{lcccc}
\hline & $2001 *$ & 2002 & 2003 & $2004 * *$ \\
\hline Neurologia / Neurocirurgia*** & 23 & 169 & 227 & 248 \\
Otorrinolaringologia & 0 & 88 & 128 & 92 \\
Terapia Intensiva & 0 & 13 & 16 & 39 \\
Cardiologia & 4 & 10 & 6 & 5 \\
Clínica geral & 0 & 2 & 3 & 8 \\
Cirurgia cardíaca & 0 & 1 & 0 & 3 \\
Cirurgia vascular & 0 & 3 & 1 & 2 \\
Pediatria & 3 & 8 & 1 & 2 \\
Outros & 0 & 4 & 5 & 6 \\
\hline
\end{tabular}

* exames realizados entre $1^{\circ}$ de agosto e 31 de dezembro de 2001; **exames realizados entre $1^{\circ}$ de janeiro e 30 de setembro de 2004.

***analisados em conjunto pelas indicações serem semelhantes.

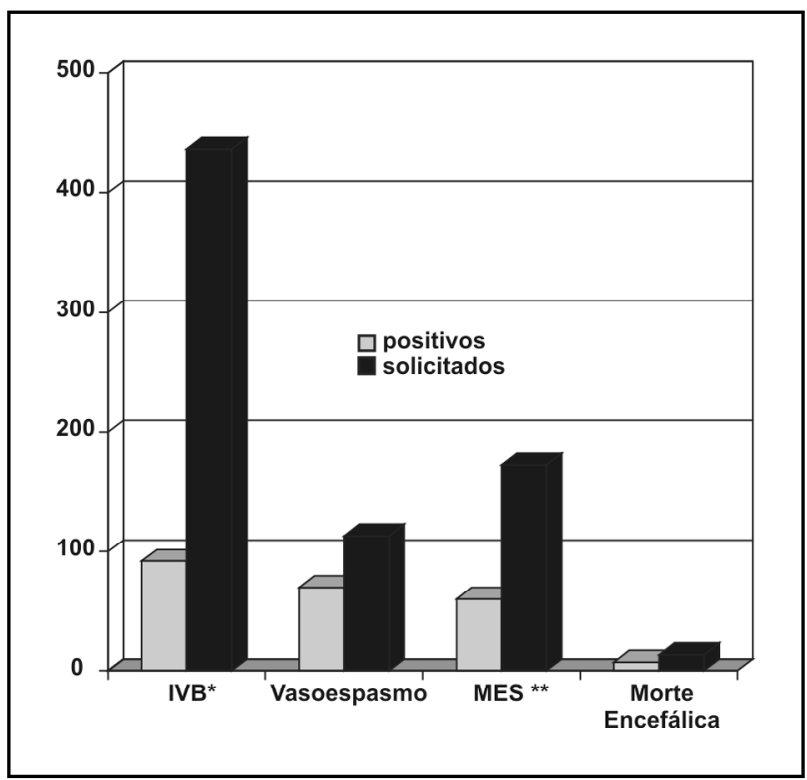

Figura. Corroboração positiva entre o DTC e a hipótese clínica. *IVB, insuficiência vertebrobasilar; **MES, microembolia. 
na estenose carotídea extracraniana, trombose venosa cerebral, migrânea, vasoespasmo secundário, pesquisa de embolia paradoxal e meningite completaram o número total de exames.

Quando correlacionamos as indicações para o exame e seus resultados, observamos que em $35 \%$ dos exames foi possível corroborar o diagnóstico positivamente, embora a exclusão da hipótese diagnóstica também seja de importância clínica. Entre as três principais solicitações para auxílio diagnóstico observamos que houve corroboração positiva da hipótese em $21 \%$ dos pacientes com insuficiência vertebrobasilar, $62 \%$ dos pacientes com vasoespasmo e $34 \%$ com fenômeno embólico em atividade (Figura). O diagnóstico de morte encefálica no nosso serviço é historicamente auxiliado pelo exame de eletrencefalograma; a solicitação de DTC ocorreu em condição específica, como nos casos de envolvimento de medicamentos supre sso res da atividade elétrica cerebral. Entre os 13 exames solicitados, 7 foram positivos.

\section{DISCUSSÃO}

Em nosso meio, observamos um aumento progressivo nas solicitações de exames de DTC nos últimos quatro anos, que ocorreu secundariamente a trabalho de divulgação das indicações e ao auxílio demonstrado pelo método nas diferentes situações clínicas onde foi aplicado. Estabelecemos ainda, linhas de pesquisa como a investigação da síndrome de insuficiência vertebrobasilar compressiva realizada em conjunto com o Serviço de Otorrinolaringologia, que marcadamente re presenta um viés nos nossos resultados $^{6}$. O estudo de reserva funcional na estenose carotídea extracraniana, apresentado como projeto de tese da própria neurologia, tem proporcionado melhor integração entre as especialidades clínica neurológica e de cirurgia vascular, redefinindo o conceito de hemodinâmica intracraniana nessa situação7. A pesquisa de embolia paradoxal por meio do DTC vem conquistando novo espaço na investigação neurológica devido tanto à simplicidade técnica de sua realização quanto à alta especificidade e sensibilidade do método. Na nossa experiência, a detecção de shunt direita-esquerda vem modificando nossa investigação em pacientes jovens com AVC isquêmico de etiologia indeterminada. Recentes publicações vêm demonstrando maior freqüência de shunt direita-esquerda em portadores de migrânea e cefaléia em salvas. Embora a fisiopatologia ainda permaneça por ser estabelecida, temos tido melhor interação entre subáreas da neurologia em nosso serviço8-10.

Obtivemos na nossa amostra concordância com a literatura quanto à dificuldade de janela de insonação, que foi de $11 \%$, visto que é descrita entre 5-15\% dos $\operatorname{casos}^{1,3}$. Observamos, no entanto, que os indícios raciais de população negra e oriental não foram os exemplos de hiperostose mais freqüentes no nosso meio, cabendo tal característica às comunidades de origem ucraniana, alemã e polonesa, de maior repre sentatividade na nossa região.

Dados de correlação positiva não são comumente levantados, visto que exames complementares necessitam de validação do método e de seu operador. Optamos por correlacionar a suspeita diagnóstica com os resultado positivos para nossa análise pessoal com intuito de saber o resultado de nossas divulgações sobre o exame. A baixa correlação de positividade do exame pode ser explicada pela exclusão da patologia suspeita com o uso do exame de DTC ou pelo desconhecimento pelo médico solicitante das indicações e utilidades do exame.

Recente publicação pela AAN estabeleceu diretrizes de utilização do exame de DTC com grau de p recisão diagnóstica e confiabilidade metodológica ${ }^{8}$. Nos últimos anos, diferentes consensos foram utilizados para determinadas investigações com o uso do DTC $4,5,11,12$. Este método vem se tornando um exame importante na prática clínica, principalmente de auxílio ao médico interessado em doenças vasculares encefálicas, possibilitando a confirmação ou excluindo em muitas situações a hipótese clínica de maneira prática e rápida. Conforme publicação recente da AAN, o DTC foi estabelecido como útil (nível A) nos seguintes tópicos: 1) identificação de risco de AVC isquêmico em crianças com anemia falciforme entre 2 e 16 anos; 2) detecção e monitoração de vasoespasmo após hemorragia subaracnóidea espontânea; 3 ) detecção de parada circulatória encefálica como exame complementar no diagnóstico de morte encefálica; 4) investigação de shunt direita-esquerda quando utilizado com agente contrastante. O DTC é provavelmente útil (nível B) em: 1) detecção de oclusão/estenose intracraniana; 2 ) estudo de vasoreatividade cerebral; 3) monitoração de trombólise cerebral no AVC agudo; 4) monitoração de procedimentos como 
endarterectomia carotídea e revascularização do miocárdo; 5) detecção de sinais de microembolia cerebral; 6) detecção e monitoração de vasoespasmo após hemorragia subaracnóidea traumática. $\mathrm{O}$ uso do DTC foi considerado possivelmente útil (nível C) na análise de estenose da artéria carótida interna extracraniana ${ }^{8}$.

\section{REFERÊNCIAS}

1. Aaslid R, Newell DW. Transcranial Doppler. New York: Raven Press, 1992: 1-8.

2. Alexandrov AV, Molina CA, Grotta JC, et al. Ultrasound-enhanced systemic thrombolysis for acute ischemic stroke. N Engl J Med 2004;351: 2170-2178.

3. Babikian VL, Wechsler LR. Transcranial Doppler ultrasonography. 2.Ed. Woburn: Butterworth-Heinemann, 1999:49-167.

4. Nichols FT, Jones AM, Adams RJ. Stroke prevention in sickle cell disease (STOP) study guidelines for transcranial Doppler testing. J Neuroimaging 2001;11:354-362.

5. Angeli S, Del Sette M, Beelke M, Anzola GP, Zanette E. Transcranial Doppler in the diagnosis of cardiac patent foramen ovale. Neurol Sci 2001;22:353-356.
6. Lange MC, Zétola VF, Muzzio JA, Nóvak EM, Mendes RC, Werneck LC. Vertebrobasilar insufficiency: the usefulness of transcranial Doppler as a complementary method (Abstr). Cerebrovasc Dis 2005;19(Suppl 1): 54.

7. Zétola VF, Massaro AR, Nóvak EM, Werneck LC, Scaff M. Is there a relationship among carotid stenosis, symptoms and cerebrovascular reactivity evaluated by the TCD with diamox test (Abstr). Cerebrovasc Dis 2005;19(Suppl 1): 54.

8. Sloan MA, Alexand rov AV, Tegeler CH, et al. Transcranial Doppler ultrasonography: report of the therapeutics and technology assessment Subcommittee of the American Academy of Neurology. Neurology 2004;62:1468-1481.

9. Del Sette M, Angeli S, Leandri M, et al. Migraine with aura and rightto-left shunt on transcranial Doppler: a case-control study. Cerebrovasc Dis 1998;8:327-330.

10. Finocchi C, Del Sette M, Angeli S, Rizzi D, Gandolfo C. Cluster headache and right-to-left shunt on contrast transcranial Doppler: a case-control study. Neurology 2004;63:1309-1310.

11. Ducrocq X, Hassler W, Moritake $K$, et al. Consensus opinion on diagnosis of cerebral circulatory arrest using Doppler-sonography: Task Force Group on cerebral death of the Neurosonology Research Group of the World Federation of Neurology. J Neurol Sci 1998;159:145-150.

12. Ringelstein EB, Droste DW, Babikian VL, et al. Consensus on microembolus detection by TCD. International Consensus Group on Microembolus Detection. Stroke 1998;29:725-729. 